\title{
Natural frequency optimization of braided bistable carbon/epoxy tubes: Analysis of braid angles and stacking sequences
}

\author{
C. $\mathrm{Wu}^{1}, \mathrm{~A}$. Viquerat ${ }^{2, *}$ \\ Faculty of Engineering and Physical Sciences, University of Surrey, GU2 7XH, United Kingdom
}

\begin{abstract}
The natural frequency of cantilevered bistable carbon/epoxy reeled composite (BRC) slit tubes constructed from combinations of braided and unidirectional (UD) plies is optimized with respect to fiber orientation angles and laminate stacking sequences. BRC tubes have the same geometry as a carpenter's tape; however, they also have a second stable configuration in the coiled state, and it is considered likely that the coiled state diameter will be fixed by the geometry of the deployment mechanism or its housing. The optimization process uses the BRC coiled diameter as a constraint, and the maximum and minimum physically achievable braid angles as bounds. Both individual tubes, and a simple deployable solar array concept are analyzed. It is observed that the braid angle, rather than ply location in the stack is of greater importance when optimizing long slender or shallow BRCs, whereas both factors must be considered in shorter BRCs. The sensitivity of natural frequency and coiled diameter to braid angle perturbations indicates the importance of precision during manufacture.
\end{abstract}

Keywords:

Natural frequency optimization, bistable composite, solar array

\section{Introduction}

Fiber braided composite materials have high specific stiffness and outstanding performance in fatigue, corrosion and thermal protective characteristics. Carbon/epoxy braided slit tubes have the same geometry as a carpenter's tape [1], but they also can be stable in a coiled state (see Figure 1(a)), compared to a carpenter's tape which is stable only when extended (Figure 1(b)). This behavior makes bistable slit tubes suitable candidates for use in a variety of deployable structures for space applications. However, one of the main challenges in using BRC tubes for very large deployable structures is maintaining a sufficiently high natural frequency. One possible application for BRC tubes in space is a deployable "roll-up" photovoltaic (PV) solar array (see Figure 2(a)) consisting of two bistable carbon-fiberreinforced polymer (CFRP) tubes and a flexible PV cell covered blanket in between. This structure essentially consists of two parallel cantilever beams side by side. If this structure is to be deployed from a spacecraft, the stiffness of the BRC tubes must be sufficiently high to avoid significant coupling between the spacecraft's control system and the solar array's structural modes. For example, Campbell [2] describes a rollable solar array design, and recommends that the natural frequency be kept above $0.2 \mathrm{~Hz}$. While the precise requirements for the vibration characteristics of a BRC based deployable structure will vary from mission to mission, it is desirable to increase the frequency of the first mode to the greatest extent possible without excessive sacrifice of performance in other areas. The natural frequency of the BRC tube supported "roll-up" solar array decreases dramatically with increase in tube length [3]. This paper presents an analysis of the effect that altering the braid fiber angles within a carbon/epoxy BRC tube has on the first "cantilever" mode, while keeping the coiled diameter of the second stable state constant. An approach to optimizing this frequency while keeping the coiled diameter constant is also given,

\footnotetext{
${ }^{*}$ Corresponding author

Email address: a.viquerat@surrey.ac.uk (A. Viquerat)

${ }^{1} \mathrm{PhD}$ Candidate

${ }^{2}$ Lecturer
} 
followed by a discussion of the sensitivity of the optimized frequency and the coiled diameter to potential small fiber misalignments encountered during manufacture.

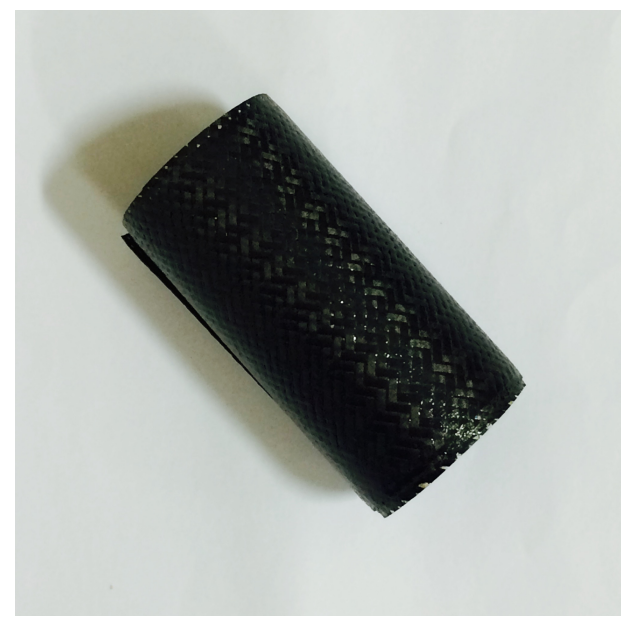

(a) Coiled stable state

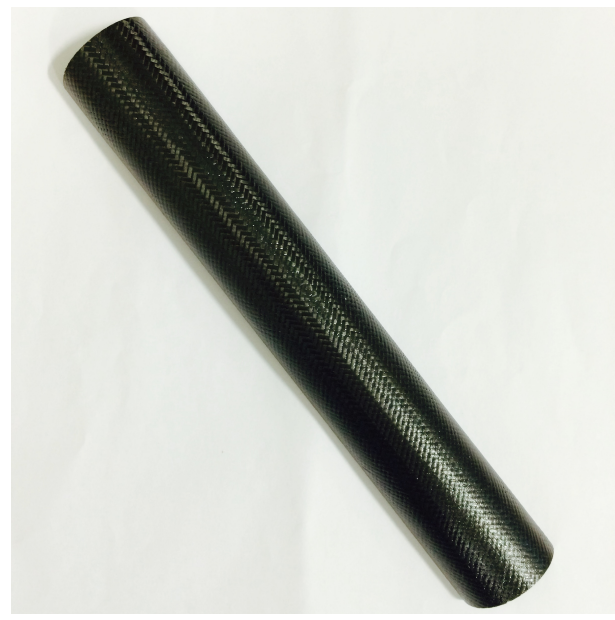

(b) Extended stable state

Figure 1: Carbon/epoxy BRC tubes at two stable configurations [4]

The maximization of the fundamental frequency of laminates has been an area of intensive research. Bert [6] first put forward the idea of maximizing the natural frequency by arranging the lamination of symmetric balanced angle-ply (SBAP) laminates on the basis of practical feasibility and manufacturing costs. Careful selection of the orientation angles of the fibers within the SBAP laminate to optimize the natural frequency was addressed in his paper. Fukunaga et al. [7] examined the optimal design of a laminate to maximize the fundamental frequencies of symmetric laminated plates on the basis of the concept of lamination parameters, which was introduced by Miki $[8,9]$ to define a set geometric properties of a laminate such as ply angles, the number of plies, stacking sequences, and unit ply thickness. A 'Layer-wise Optimization Approach' (LOA) for maximizing the fundamental frequencies of laminated composite plates or shallow cylindrical shells has also been proposed [10,11]. This approach took the orientation angles of fibers in each ply as variables and utilized an iterative procedure to find a solution for the optimal natural frequency of the entire laminate. Betts [12] defined an optimization method using stability as a constraint, with an objective function based on a ratio of laminate stiffness in two specified directions. More research related to the dynamic response of shells can be found in literature [13, 14, 15]. Note, however, that very few of these publications discuss the effect of uncertainties or small perturbations in fiber angles within the composite laminate.

The effects of other types of uncertainty in the mechanical response of composite structures has been analyzed in a number of articles $[16,17,18]$. The sensitivity of bistable laminates has also been examined [19]. The main sources of uncertainty in composite materials [20,21,22, 23] include variability in material properties due to indeterminate fiber and matrix properties, geometric aspects at macroscopic level, and the manufacturing process itself [24]. The dynamic response of structures with uncertainties in the composite material was studied using a parametric probabilistic approach by assigning random variables to certain parameters [25]. The uncertainty in fiber angles in each ply of a composite laminate is an important concern in the dynamic response of BRC tubes.

This paper presents an analysis of natural frequency and coiled diameter with respect to braid fiber angles. The nonlinear constrained vibration optimization procedure $[26,4]$ combining a Finite Element (FE) numerical model in FE commercial code ABAQUS [27] with Matlab [28] optimization functions is briefly described. A stability margin constraint, the natural frequency and the coiled diameter are studied for different laminate stacking sequences. A deployable "roll-up" solar array with no PV membrane is also modeled in ABAQUS for the purpose of validating the feasibility of analyzing one single BRC tube instead of the whole solar array structure. The natural frequencies of several BRC tubes with various dimensions, constraints and stacking sequences are optimized with respect to 


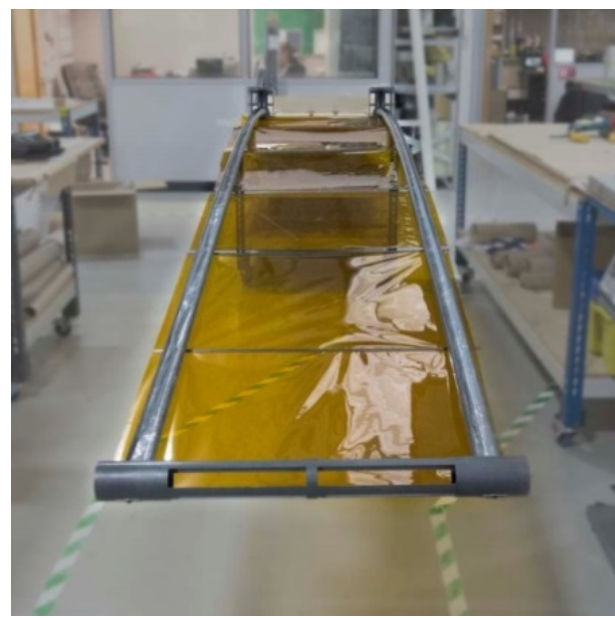

(a) Deployable "roll-up" PV solar array developed for DeployTech [5]

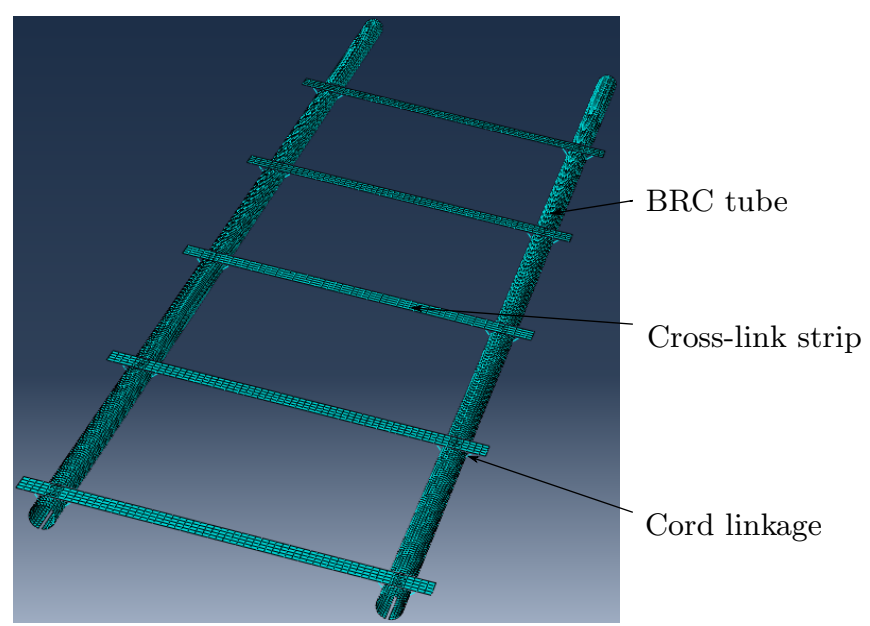

(b) Assembly of a deployable "roll-up" solar array with no PV blanket in ABAQUS

Figure 2: Deployed "roll-up" PV solar array

braid angles within the laminates. The effects of longitudinal and transverse stiffness on the natural frequency of a slender BRC tube are computationally investigated. Finally, concluding remarks are outlined.

\section{Model development}

\subsection{Description of a BRC tube model}

A simple sketch of the BRC tube cross-section is shown in Figure 3. The BRC tube model is based on the following assumptions:

1. Classical laminate theory (CLT) [29] is sufficient to capture the mechanical behavior of the tubes.

2. A single carbon/epoxy braid ply is modelled as two UD plies, each taking half of the properties.

3. All braid plies have the same material properties.

4. The BRC tube properties do not change along the length (no change in braid angles, volume fractions or number of plies).

5. The braid angles are measured from the longitudinal $(z)$ axis.

Braided fibers are often used in the production of BRCs because it is possible to produce extremely long continuous plies with non-zero fiber angles, and because it is easy to set the fiber angles to almost any value desired during manufacture.

\subsection{Analytical bistability model}

An analytical model developed by Guest and Pellegrino [30] was employed to model the bistability of the tubes because of the simplicity of its implementation, and because it has previously proved to be accurate in predicting the second stable state coiled diameters of the types of BRC tubes used in this study [31]. It is assumed that the mid-surface of the BRC tube experiences no stretching, and also that the bending experienced by the tube is uniform everywhere. The coiled diameter of the second stable state is found as [30] :

$$
D_{c}=2 * \frac{D_{11}}{D_{12}} * R
$$




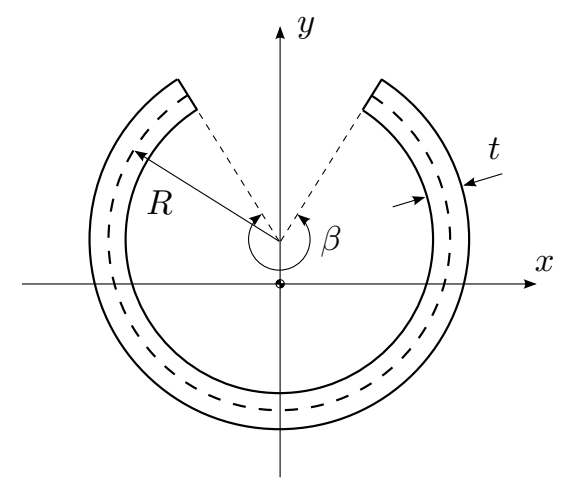

Figure 3: BRC tube cross-section. $\beta$ is the included angle, $t$ is the tube thickness, $R$ denotes the initial radius measured to the midplane of the tube wall, and $x$ and $y$ are the axes of the cross-section

where $R$ is the initial radius of the tube, and $D_{i j}$ is the $i, j^{\text {th }}$ element in the bending stiffness matrix of CLT [29].

Guest's model also provides a quantitative measure of the extent to which the coiled configuration of a BRC tube can be expected to be stable:

$$
S=4 * \hat{D}_{66}+2 * \hat{D}_{12}-2 * \frac{\hat{D}_{22}}{\hat{D}_{12}}
$$

where $\hat{D}_{i j}=D_{i j} / D_{11}$.

Equation 2 can be used to determine whether two separate stable configurations exist, and Equation 1 can be used to calculate the theoretical coiled diameter of the BRC tube at the second stable state. These two equations are employed in the natural frequency optimization loop to ensure that a specified coiled diameter is kept constant, while a sufficient level of stability is also maintained. A second stable equilibrium only exists when the sign of Equation 2 is positive. Thus, $S>0$ is used as a stability criterion.

\subsection{Finite element model}

For most BRC geometries, the fundamental frequency of a deployed tube used in the solar array with fixed-free boundary conditions most likely corresponds to a simple up-down "cantilever" mode in which the motion of the tube tip occurs only within the $y-z$ (see Figure 3) plane [32]. Note that the lowest frequency mode of a single BRC may, in fact, be a side-to-side, rather than up-down cantilever mode: see Section 3. The deployable "roll-up" PV solar array (as shown in Figure 2(a)) was studied in previous papers [4, 26]. The natural frequency of a deployed BRC can, in some cases, be predicted with accuracy using a simple beam model. However, to ensure accuracy across a range of BRC geometries a finite element (FE) method was used to perform the modal analysis: first on a deployed solar array structure, then on individual extended BRC tubes. A finite element approach like this has been used for this type of modal analysis in the past [3]. The composite model was implemented in ABAQUS using a linear perturbation approach to compute the free vibration frequencies of extended fixed-free BRC tubes with the Lanczos algorithm available in ABAQUS/standard solver [33]. The fiber angle of each ply is used to compute the lamina stiffness ([ABD] matrix [29]). Larger-strain shell element S4R, as a robust quadrilateral element for general-purpose applications, was selected to mesh the tube model. The mesh density and total number of element varied with each case.

\subsection{Solar array model}

A deployed "roll-up" PV solar array (see Figure 2(a)) was modeled in ABAQUS (Figure 2(b)) to capture the lowest frequency vibration mode. The model consists of two fixed-free BRC tubes rigidly connected to each other using five $80 \mathrm{~mm}$ wide and $1200 \mathrm{~mm}$ long discrete rigid shell planar strips with a mass $0.1 \mathrm{~kg}$ of each. These strips are spaced at $1 \mathrm{~m}$ intervals along the length of the array, starting $100 \mathrm{~mm}$ in from the free end of the solar 
array. High tensile elastic $\left(\rho=1000 \mathrm{~kg} / \mathrm{m}^{3}, E=20 \mathrm{GPa}, \nu=0.3\right)$ cord links with a radius of $1 \mathrm{~mm}$ connect the strips to the BRCs. These are used to prevent rotation of the BRCs with respect to the strips in the deployed solar array FE model [5]. The blanket is not included in this FE analysis because it would obscure the modes of interest by introducing many extremely low frequency modes generated by the soft flexible blanket substrate material. Each BRC is constructed using four-node reduced integration shell (S4R) elements. The cross-link strips (see strips connecting the two parallel BRCs in Figure 2(b)) are meshed with a four-node three dimensional bilinear rigid quadrilateral element (R3D) [27]. The linkage cords are modeled as beam elements (B31). The simulation verified that the lowest frequency of the complete structure corresponds to the "cantilever" mode, making this the vibration mode of greatest interested in this article.

\subsection{Vibration optimization}

The lamination parameter approach is often used in optimization studies as any stacking sequence can be represented by no more than twelve lamination parameters. Some solutions include fractional ply numbers, however, it is possible to obtain practical sets of parameters by choosing physically obtainable values nearby to those produced by the initial lamination parameter optimization. Wu et. al. [34] developed an optimization process for practical design and manufacturing purposes in which a secondary optimization process uses the mean of the least square distances between the obtained and target lamination parameters evaluated at various points on the laminate plate. Fractional plies can also be rounded up to the nearest obtainable integer number, resulting in the most lightweight feasible design. The lamination parameter approach requires that the ply material remain the same, and that the material properties do not vary spatially over the structure. In this article, the braided BRC tubes analyzed must be extremely flexible, so even with very thin plies, BRC tubes rarely consist of more than a combination of six to eight unidirectional and braided plies. For practical purposes, only BRC tubes with fewer than six plies are considered here, with an optimization procedure operating directly on ply fiber angle used.

The BRC consists of a number of braided plies with angles $\alpha_{i}$, and a number of unidirectional plies oriented with the fibers along the longitudinal axis $\left(0^{\circ}\right)$. The braid angles are taken as the optimization variables, and the coiled diameter is set as a constraint. The braid angles must remain between bounds representing the physically achievable limits of the particular braid used. The coiled diameter is set as a constraint because it is considered likely that this parameter will be fixed by the geometry of the deployment mechanism or its housing. A stability criterion is set as an inequality constraint to ensure the existence of a second stable equilibrium. The optimization formulation is:

$$
\begin{array}{ll}
\text { Maximize: } & \text { Natural frequency of a BRC tube } \\
\text { Variables: } & \alpha_{i} \\
\text { Subject to: } & \alpha_{i \min } \leq \alpha_{i} \leq \alpha_{i \max } \\
& D_{c}\left(\alpha_{i}\right)=D_{c \text { specified }} \\
& -S \leq 0
\end{array}
$$

The constrained nonlinear optimization is illustrated in Figure 4. This process could be applied to any number of braid plies, while it is possible to use the braid angle of every ply in the laminate as an independent optimization variable, the same variable can be assigned to more than one ply if necessary to simplify the optimization process, and take advantage of obvious symmetries in the laminate. Most BRCs considered here are assigned two independent variables (Tables 2 and 3), with one four-ply example considered later (Table 6).

The model described in this section is limited in its ability to accurately model particularly shallow tubes, as well as tubes with high curvatures either in their deployed or stowed configurations. A more detailed micro-mechanical model of the braided plies would improve the accuracy of the optimization (for example by capturing the fibre microbuckling behavior at higher tube curvatures). Guest's model is also limited in its ability to incorporate higher curvatures as it is also based on CLT and neglects through-thickness shear, edge effects, and the influence of included angle on the behavior of the bistable tube. 


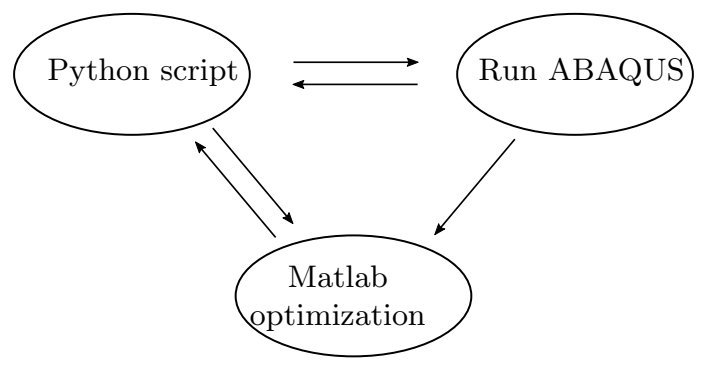

Figure 4: Nonlinear constrained optimization loop, in which a numerical model of an extended BRC tube is created in ABAQUS using a Python script. The direct search solver patternsearch in the Matlab global optimization toolbox [35] is then employed to optimize the natural frequency, updating the BRC parameters again via Python. The non-gradient-based patternsearch evaluates the objective function at a series of points in a mesh, and then uses the location of the point with the best objective value to guide the next step in the search. Function tolerance, step tolerance and constraint tolerance are all set to $1 \times 10^{-6}$ in the solver options.

\section{Results and Discussion}

The FE method was applied to BRC tubes with different ply sequences, as well as different geometries $(R$ and $\beta$ values). The material properties of the carbon/epoxy braid and unidirectional (UD) plies are given in Table 1 [36]. The maximum and minimum allowable braid angles are taken to be $75^{\circ}$ and $15^{\circ}$ [37], respectively.

Table 1: Material properties of half-carbon/epoxy-braid and UD plies

\begin{tabular}{ccccccccc}
\hline \hline Materials & $E_{1}(\mathrm{GPa})$ & $E_{2}(\mathrm{GPa})$ & $\nu_{12}$ & $G_{12}(\mathrm{GPa})$ & $G_{13}(\mathrm{GPa})$ & $G_{23}(\mathrm{GPa})$ & $t(\mathrm{~mm})$ & $\rho\left(\mathrm{kg} / \mathrm{m}^{3}\right)$ \\
\hline $\pm 45^{\circ}$ half-braid ply & 66.81 & 3.705 & 0.278 & 2.471 & 2.471 & 2.471 & 0.046 & 1480 \\
\hline UD ply & 77.372 & 4.9 & 0.309 & 3.293 & 3.293 & 3.293 & 0.057 & 1000 \\
\hline \hline
\end{tabular}

A solar array FE model described in Section 2.4 consisting of two BRCs of Tube 1 type, and with geometric properties given in Table 2 was modeled initially. The natural (cantilever mode) frequency of the solar array is plotted as a function of braid angles in Figure 5(a). The dynamic response of a single BRC is presented for comparison in Figure 5(b) in order to verify the feasibility of analyzing an individual tube instead of a complete solar array structure. It is important to note that the cantilever mode for a single Tube 1 type BRC presents as the second lowest frequency mode, rather than the first (the lowest is a side-to-side swaying motion, not present in the solar array structure). Hence, Figure 5(b) presents the second mode for this BRC. Comparing the two figures illustrates great similarity, although the values of solar array's frequencies are slightly lower than those of the single tube due to the mass addition of the strips and rigid cord linkages. The inclusion of a flexible PV blanket in the model would decrease this frequency further. Nevertheless, the model creation and computing cost of the entire solar array structure is higher than simply modeling one tube. Thus, it is efficient and reasonable to analyze the dynamic behavior of a single tube, rather than that of the entire solar array structure. The following analysis will be conducted for individual BRC tubes.

To understand the effect of varying the braid angles on the coiled diameter, the bistability, and the cantilever frequency of BRC tubes at the extended configuration, BRC tubes (see Table 2) with different laminate stacking sequences were chosen for the analysis. Their properties are investigated over the entire allowable braid angle range. Contour plots illustrating the cantilever frequency, coiled diameter and stability constraint as functions of braid angles are shown in Figure 6. For the same $\alpha_{1}$ and $\alpha_{2}$, the cantilever frequency of Tube 2 is always higher than Tube 1 and Tube 3 due to the introduction of a UD ply, while Tube 1 only has slightly higher cantilever frequency than Tube 3 due to the mass addition accompanying the number of plies increase.

As shown in Figure 6, the frequency contours for Tube 1, Tube 2 and Tube 3 are symmetric about $\alpha_{1}=\alpha_{2}$. In 


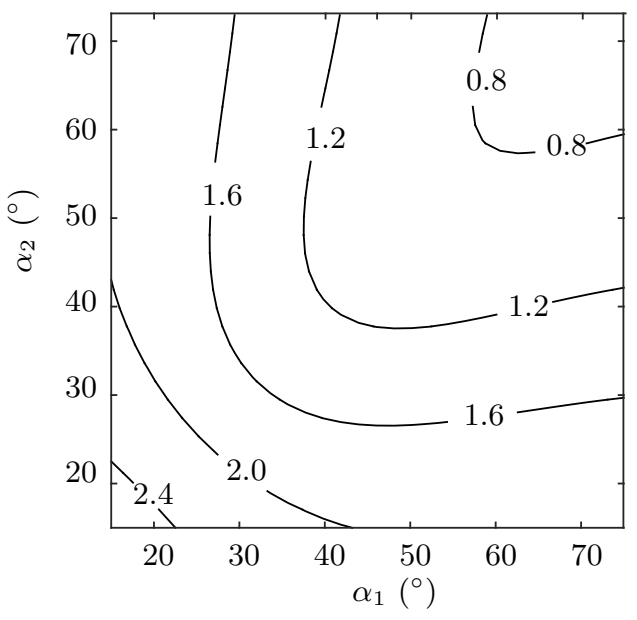

(a) A deployed solar array without PV blanket

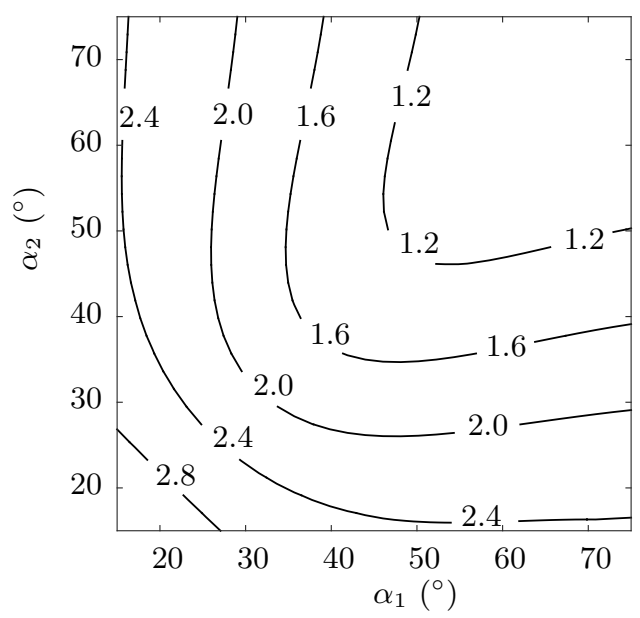

(b) A single Tube 1 from Table 3

Figure 5: Contour plots illustrating cantilever mode frequencies (black lines) as a function of braid angles. Figure (a) is for a $5.1 \mathrm{~m}$ long deployed solar array described in Section 2.4 with two rigidly connected Tube 1 type BRCs from Table 3, while Figure (b) is for the single Tube 1. The similarity of Figure (a) and Figure (b) implies the reliability of analyzing a single BRC tube instead of a complete solar array structure, which will be validated in Section 3.1

Table 2: Laminate specifications and geometry of BRC tubes used to investigate the effects of braid angles within different laminate stacking sequences. The number of mesh element is for individual tube model in ABAQUS

\begin{tabular}{|c|c|c|c|c|c|}
\hline \multirow[b]{2}{*}{$\begin{array}{l}\text { Tube } \\
\text { No. }\end{array}$} & \multirow[b]{2}{*}{ Stacking sequence } & \multicolumn{3}{|c|}{ BRC tube properties } & \multirow[b]{2}{*}{$\begin{array}{l}\text { No. of S4R } \\
\text { elements }\end{array}$} \\
\hline & & $\begin{array}{l}\text { Length } \\
(\mathrm{m})\end{array}$ & $\begin{array}{l}R \\
(\mathrm{~mm})\end{array}$ & $\beta\left({ }^{\circ}\right)$ & \\
\hline 1 & {$\left[ \pm \alpha_{1} / \pm \alpha_{2} / \pm \alpha_{2} / \pm \alpha_{1}\right]$} & 5.1 & 38 & 345 & 3000 \\
\hline 2 & {$\left[ \pm \alpha_{1} / \pm \alpha_{2} / 0 / \pm \alpha_{2} / \pm \alpha_{1}\right]$} & 5.1 & 38 & 345 & 3000 \\
\hline 3 & {$\left[ \pm \alpha_{1} / \pm \alpha_{2}\right]$} & 5.1 & 38 & 345 & 3000 \\
\hline
\end{tabular}

other words, the $\alpha_{1}$ and $\alpha_{2}$ plies are interchangeable (see Table 4) and the optimal point for each individual tube can be estimated combining the coiled diameter and stability constraints. To investigate the ply location's effect further, the $\alpha_{1}$ and $\alpha_{2}$ braid plies within laminates of Tube 1 (Table 2), Tube 4 and Tube 6 (Table 3) are swapped. The results are given in Table 4, where the natural frequency variation can be seen to be very small for slender or shallow tubes (Tube 1 and Tube 6), but much larger for the short and deep tube (Tube 4). The coiled diameter is significantly affected by changing the ply locations for all tubes.

The cantilever frequency contours as illustrated in Figure 6, are symmetric in $\alpha_{1} / \alpha_{2}$ for long BRC tubes with different laminate stacking sequences. In particular, for Tube 3 the natural frequency, coiled diameter and stability contours are all symmetric about $\alpha_{1}=\alpha_{2}$ (see Figure 6(c)), which implies two optimal solutions for this special tube. For the purpose of achieving a better understanding of the effects of braid angle variation on the dynamic response of extended BRC tubes, a set of BRCs with different geometric properties (given in Table 3) were analyzed. The shorter tubes show a greater variety of modal shapes at the lower end of the frequency scale, occasionally making the cantilever mode difficult to distinguish. This may be due to the laminate longitudinal and flexural stiffness change with combinations of $\alpha_{1}$ and $\alpha_{2}$, which is investigated in Section 3.2. The contour plots of cantilever frequency as a function of $\alpha_{1}$ and $\alpha_{2}$ in Figures 7(a) and 7(b) indicate that the dynamic response of shorter tubes is not symmetric in $\alpha_{1} / \alpha_{2}$, as those of longer tubes appears to be. This is because transverse bending stiffness $\left(D_{22}\right)$ plays a larger role in determining the cantilever frequency in short tubes due to cross-sectional deformation (see Figure 


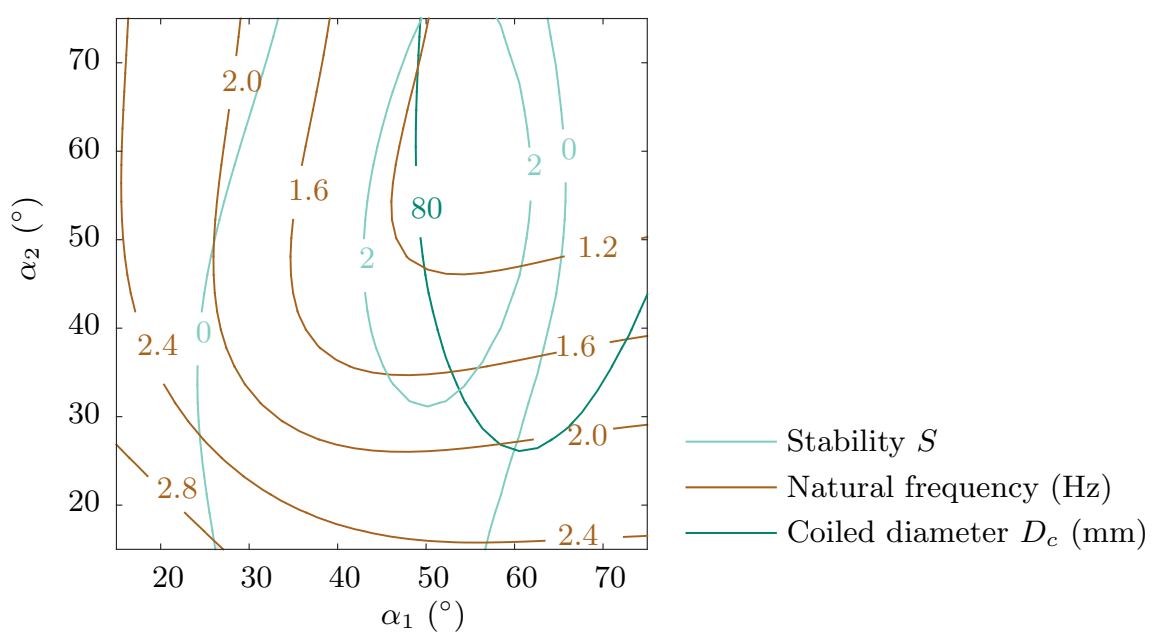

(a) Tube 1

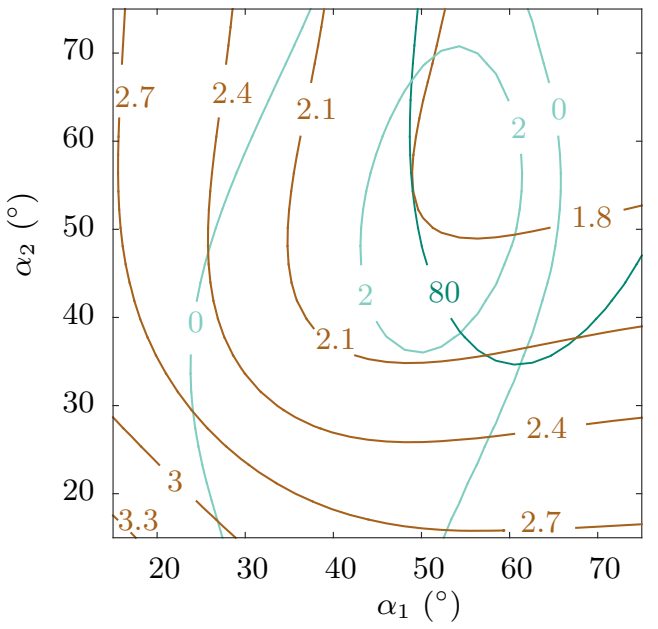

(b) Tube 2

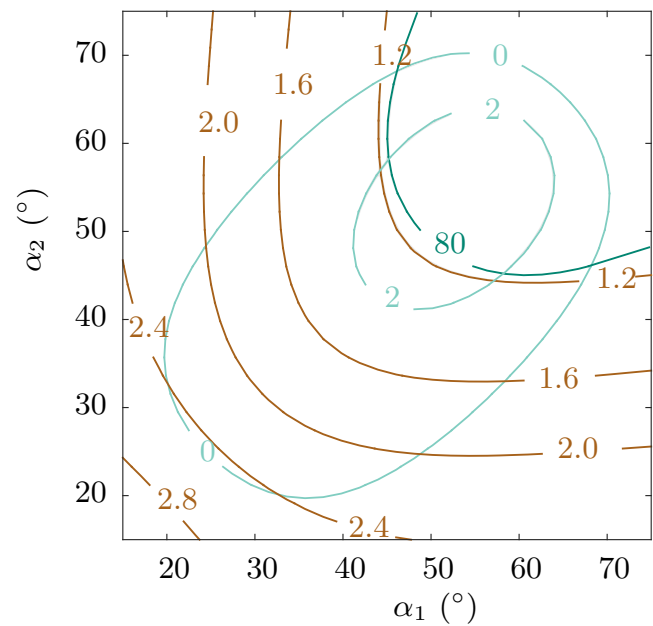

(c) Tube 3

Figure 6: Contour plots of cantilever frequency, stability and coiled diameter as functions of braid angles for BRCs in Table 2. For each case, only a single coiled diameter contour $\left(D_{c}=80 \mathrm{~mm}\right)$ is shown for clarity. The region enclosed by the $S=0$ contour indicates the range of braid angles for which a second stable state exists.

$7(\mathrm{~d}))$ effects [22, 38], especially in those tubes with larger included angles. Reducing the included angle restores this symmetry to a large extent (see Figure $7(\mathrm{c})$ ). The mismatch between the clamped boundary condition at one end of the tube and the free condition at the other is more noticeable in the shorter tubes. The asymmetry of frequency contours in Figure 7(a) and Figure 7(b) indicates that the locations of the $\alpha_{1}$ and $\alpha_{2}$ plies within the laminate stack will impact the natural frequency and vibration modes of relatively short tubes.

\subsection{Braid angle optimization}

This section describes the results of the constrained optimization of the cantilever frequency of a number of fixed-free extended BRC tubes. The optimized braid angles for each laminate stacking sequence are given in Table 5. Comparing the results for Tube 1, Tube 2 and Tube 3 shows that the same coiled diameter can be achieved using a variety of different laminate layups. The fact that the optimization results for Tube 1 and Tube 4 yield the same set of braid angles implies that the tube length has little effect on the braid angle optimization. Tubes 4 and Tube 5 illustrate that the radius $R$ of a tube impacts the optimization results regarding to the coiled diameter constraint. Tubes 5 and 9 from Table 6 yield extremely similar optimal solutions, implying the symmetry of plies 
Table 3: Laminate specifications, geometries and number of mesh element in ABAQUS for short BRC tubes. They are proposed to study the effects of tube geometric properties on the vibration performance

\begin{tabular}{llllll}
\hline \hline & & \multicolumn{3}{c}{ BRC tube properties } \\
$\begin{array}{l}\text { Tube } \\
\text { No. }\end{array}$ & Stacking sequence & $\begin{array}{l}\text { Length } \\
(\mathrm{m})\end{array}$ & $R$ & $\beta\left(^{\circ}\right)$ & $\begin{array}{l}\text { No. of S4R } \\
\text { elements }\end{array}$ \\
\hline 4 & {$\left[ \pm \alpha_{1} / \pm \alpha_{2} / \pm \alpha_{2} / \pm \alpha_{1}\right]$} & 1.0 & 38 & 345 & 1500 \\
\hline 5 & {$\left[ \pm \alpha_{1} / \pm \alpha_{2} / \pm \alpha_{2} / \pm \alpha_{1}\right]$} & 1.0 & 25 & 345 & 1500 \\
\hline 6 & {$\left[ \pm \alpha_{1} / \pm \alpha_{2} / \pm \alpha_{2} / \pm \alpha_{1}\right]$} & 1.0 & 38 & 137.5 & 1000 \\
\hline 7 & {$\left[ \pm \alpha_{1} / \pm \alpha_{2} / \pm \alpha_{2} / \pm \alpha_{1}\right]$} & 1.0 & 25 & 353 & 1500 \\
\hline \hline
\end{tabular}

Table 4: Effect of swapping $\alpha_{1}$ and $\alpha_{2}$ plies within a laminate stacking sequence $\left[ \pm \alpha_{1} / \pm \alpha_{2} / \pm \alpha_{2} / \pm \alpha_{1}\right]$ on their natural frequencies and coiled diameters

\begin{tabular}{|c|c|c|c|c|c|c|c|}
\hline \multirow[b]{2}{*}{ Tube label } & \multicolumn{3}{|c|}{ BRC tube properties } & \multicolumn{2}{|c|}{ Braid angles } & \multirow[b]{2}{*}{$\begin{array}{l}D_{c} \\
(\mathrm{~mm})\end{array}$} & \multirow[b]{2}{*}{$\begin{array}{l}\mathrm{NF} \\
(\mathrm{Hz})\end{array}$} \\
\hline & $\begin{array}{l}\text { Length } \\
(\mathrm{m})\end{array}$ & $\begin{array}{l}\mathrm{R} \\
(\mathrm{mm})\end{array}$ & $\beta(0)$ & $\alpha_{1}\left(^{\circ}\right)$ & $\alpha_{2}\left(^{\circ}\right)$ & & \\
\hline$S_{1}$ & 5.1 & 38 & 345 & 60 & 26 & 80 & 2.15 \\
\hline$S_{2}$ & 5.1 & 38 & 345 & 26 & 60 & 303.20 & 2.15 \\
\hline$S_{3}$ & 5.1 & 38 & 345 & 55 & 35 & 75.42 & 1.7 \\
\hline$S_{4}$ & 5.1 & 38 & 345 & 35 & 55 & 168.06 & 1.7 \\
\hline$S_{5}$ & 1.0 & 38 & 345 & 60 & 26 & 52.49 & 28.36 \\
\hline$S_{6}$ & 1.0 & 38 & 345 & 26 & 60 & 198.95 & 19.82 \\
\hline$S_{7}$ & 1.0 & 38 & 137.5 & 60 & 26 & 80 & 8.51 \\
\hline$S_{8}$ & 1.0 & 38 & 137.5 & 26 & 60 & 303.20 & 8.21 \\
\hline
\end{tabular}

within the laminate and the efficiency of optimizing two variables instead of four. Tubes 1 and $1_{\text {shallow }}$ have identical braid angles, suggesting that the included angle also has little effect on the braid angle optimization. The stability constraint is active for Tubes $1,2,1_{\text {shallow }}$ and 4 , and inactive for the others. Tube 7 have active braid angle bound constraints. However, Tube 3, consisting of only two braid plies, does not have active constraints at its optimum. This can be verified through Figure 6(c), where frequency, coiled diameter and stability contours are all symmetric with reference to $\alpha_{1} / \alpha_{2}$. The coiled diameter contour (at $D_{c}=80 \mathrm{~mm}$ ) becomes parallel to sets of frequency contours (potential optimal points) at two pairs of $\alpha_{1}$ and $\alpha_{2}$ values $\left(\left[\alpha_{1}, \alpha_{2}\right]=[60,45]\right.$ and $\left.\left[\alpha_{1}, \alpha_{2}\right]=[45,60]\right)$. Both pairs are located within the stability constrained region.

Multiple initial starting points for the same model converge on the same optimal point, and the local area around each optimal points was inspected visually using $2 \mathrm{D}$ slices or contour plots to confirm the nature of the stationary point.

For the purpose of validating the optimization results with the complete solar array model, the optimal braid angles are substituted into both individual tube and solar array models. The results are given in Table 7 . The agreement between natural frequency values for the complete solar array and corresponding individual BRC tubes indicates that the optimized solutions for a single tube model are applicable to the complete model.

\subsection{Effect of $A_{11}, D_{11}$ and $D_{c}$ on cantilever mode frequency}

In some cases a good approximation to the natural frequency of an extended BRC can be obtained by taking the $A_{11}$ term for longitudinal stiffness, and using it to estimate the first vertical bending mode from Euler-Bernoulli 
Table 5: Cantilever frequency optimization of uniform BRC tubes with coiled diameter and stability constraints

\begin{tabular}{llllllllll}
\hline \hline Tube & Stacking sequence & $\begin{array}{l}\text { Length } \\
\text { ThC tube properties }\end{array}$ & $\beta$ & \multicolumn{3}{l}{ Optimized braid angles } \\
No. & & $(\mathrm{m})$ & $(\mathrm{mm})$ & & $\begin{array}{l}D_{c} \\
(\mathrm{~mm})\end{array}$ & $\alpha_{1}\left(^{\circ}\right)$ & $\alpha_{2}\left(^{\circ}\right)$ & $\begin{array}{l}\text { NF } \\
(\mathrm{Hz})\end{array}$ & $\begin{array}{l}\text { Stability } \\
S\end{array}$ \\
\hline 1 & {$\left[ \pm \alpha_{1} / \pm \alpha_{2} / \pm \alpha_{2} / \pm \alpha_{1}\right]$} & 5.1 & 38 & 345 & 80 & 60.0 & 26.0 & 2.15 & 0 \\
\hline 2 & {$\left[ \pm \alpha_{1} / \pm \alpha_{2} / 0 / \pm \alpha_{2} / \pm \alpha_{1}\right]$} & 5.1 & 38 & 345 & 80 & 61.0 & 34.5 & 2.27 & 0 \\
\hline 3 & {$\left[ \pm \alpha_{1} / \pm \alpha_{2}\right]$} & 5.1 & 38 & 345 & 80 & 60.0 & 45.0 & 1.24 & 1.70 \\
\hline $1_{\text {shallow }}\left[ \pm \alpha_{1} / \pm \alpha_{2} / \pm \alpha_{2} / \pm \alpha_{1}\right]$ & 5.1 & 38 & 262 & 80 & 60.0 & 26.0 & 1.64 & 0 \\
\hline 4 & {$\left[ \pm \alpha_{1} / \pm \alpha_{2} / \pm \alpha_{2} / \pm \alpha_{1}\right]$} & 1.0 & 38 & 345 & 80 & 60.0 & 26.0 & 28.35 & 0 \\
\hline 5 & {$\left[ \pm \alpha_{1} / \pm \alpha_{2} / \pm \alpha_{2} / \pm \alpha_{1}\right]$} & 1.0 & 25 & 345 & 80 & 46.5 & 15.5 & 36.98 & 1.19 \\
\hline 7 & {$\left[ \pm \alpha_{1} / \pm \alpha_{2} / \pm \alpha_{2} / \pm \alpha_{1}\right]$} & 1.0 & 25 & 353 & 65 & 54.0 & 15.0 & 38.51 & 0.61 \\
\hline \hline
\end{tabular}

Table 6: Cantilever frequency optimization of a uniform BRC tube having the same geometric properties as Tube 5 from Table 5, but with an independent variable for each ply angle

\begin{tabular}{lllllllll}
\hline \hline & & \multicolumn{7}{c}{ Optimized braid angles } \\
$\begin{array}{l}\text { Tube } \\
\text { No. Stacking sequence }\end{array}$ & $D_{c}(\mathrm{~mm})$ & $\alpha_{1}\left({ }^{\circ}\right)$ & $\alpha_{2}\left({ }^{\circ}\right)$ & $\alpha_{3}\left(^{\circ}\right)$ & $\alpha_{4}\left(^{\circ}\right)$ & NF & Stability \\
\hline 9 & {$\left[ \pm \alpha_{1} / \pm \alpha_{2} / \pm \alpha_{3} / \pm \alpha_{4}\right]$} & 80 & 46.0 & 15.0 & 15.0 & 47.0 & 37.1 & 1.16 \\
\hline \hline
\end{tabular}

beam theory (Equation 3).

$$
\omega=\frac{1.8751^{2}}{L^{2}} \sqrt{\frac{E I}{A \rho}}
$$

where $\omega$ is the vibration frequency, $L$ represents the tube length, $E$ denotes the Young's modulus, $I, A$ and $\rho$ are the second moment of area, cross-section area and material density, respectively. For a composite, the Young's modulus can be estimated using the longitudinal stiffness $A_{11}$ and flexural stiffness $D_{11}$. However, the laminates modeled herein are so thin that the bending stiffness of the tube is determined almost entirely by the geometry of the section and the longitudinal stiffness of the laminate. As the included angle is decreased, the $D_{11}$ term will have more of an effect, however, it is still remarkably small. For example, with $A_{11}=1.0869 \times 10^{7} \mathrm{~N} / \mathrm{m}$, $D_{11}=0.0686 \mathrm{Nm}, \beta=353^{\circ}, R=25 \mathrm{~mm}$, and a thickness of $0.368 \mathrm{~mm}$, a tube bending stiffness of $512.397 \mathrm{~N} / \mathrm{m}^{2}$ is obtained without including $D_{11}$, and $512.402 \mathrm{~N} / \mathrm{m}^{2}$ including it. Decreasing the included angle to $30^{\circ}$, bending stiffnesses of $0.0092 \mathrm{~N} / \mathrm{m}^{2}$ and $0.0101 \mathrm{~N} / \mathrm{m}^{2}$ are determined respectively. Thus, the beam theory bending stiffness in this article is calculated only using the $A_{11}$ term of the ABD matrix [39]. After using Equation 3 initially in the frequency optimization process, it was discovered that the $A_{11}$ term is not always sufficient to fully capture the modal behavior of an extended BRC, particularly for short BRC tubes with deep included angle. In this section, the stiffness terms $A_{11}$ and $D_{11}$ are investigated to determine their individual effects on the cantilever frequency. An FE model is used to determine the modal frequencies. Tube 7 from Table 5 was chosen for the investigation. 
Table 7: Validation of the braid angle optimization results for the complete solar array model. Three different BRCs are considered.

\begin{tabular}{llllll}
\hline \hline \multirow{2}{*}{ Model name } & Stacking sequence & \multicolumn{4}{c}{ BRC tube properties } \\
& & $\begin{array}{l}\text { Length } \\
(\mathrm{m})\end{array}$ & $\begin{array}{l}R \\
(\mathrm{~mm})\end{array}$ & $\beta\left(^{\circ}\right)$ & $\mathrm{NF}$ \\
& & 5.1 & 38 & 345 & 2.15 \\
\hline Single tube 1 & {$[ \pm 60 / \pm 26 / \pm 26 / \pm 60]$} & 5.1 & 38 & 345 & 2.06 \\
\hline Solar array 1 & {$[ \pm 60 / \pm 26 / \pm 26 / \pm 60]$} & 38 & 345 & 2.27 \\
\hline Single tube 2 & {$[ \pm 61 / \pm 34.5 / 0 / \pm 34.5 / \pm 61]$} & 5.1 & 38 & 3.17 \\
\hline Solar array 2 & {$[ \pm 61 / \pm 34.5 / 0 / \pm 34.5 / \pm 61]$} & 5.1 & 38 & 345 & 2.17 \\
\hline Single tube 3 & {$[ \pm 60 / \pm 45]$} & 5.1 & 38 & 345 & 1.24 \\
\hline Solar array 3 & {$[ \pm 60 / \pm 45]$} & 5.1 & 38 & 345 & 1.20 \\
\hline \hline
\end{tabular}

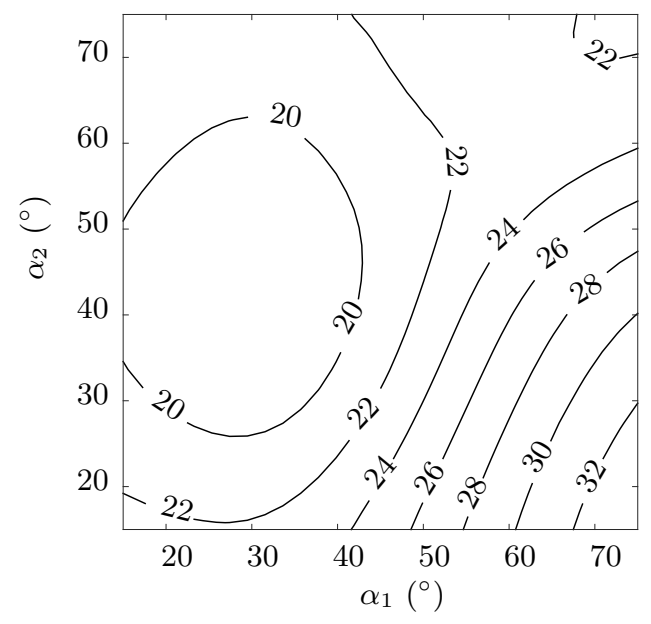

(a) Tube 4

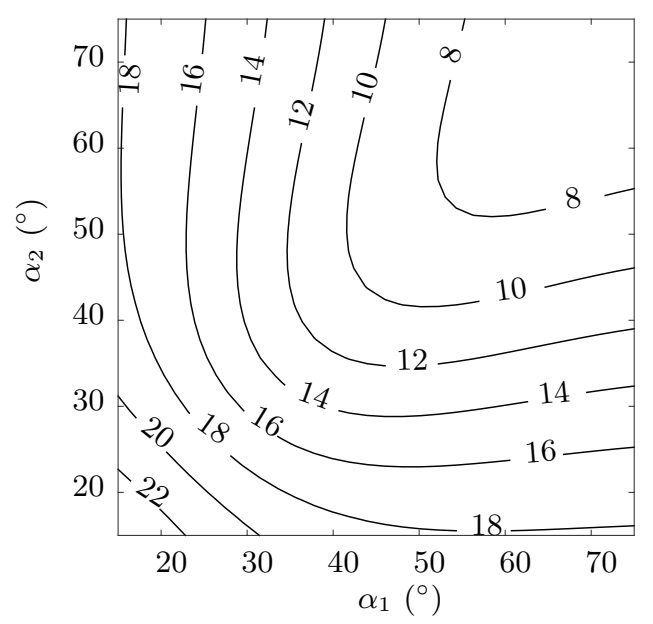

(c) Tube 6

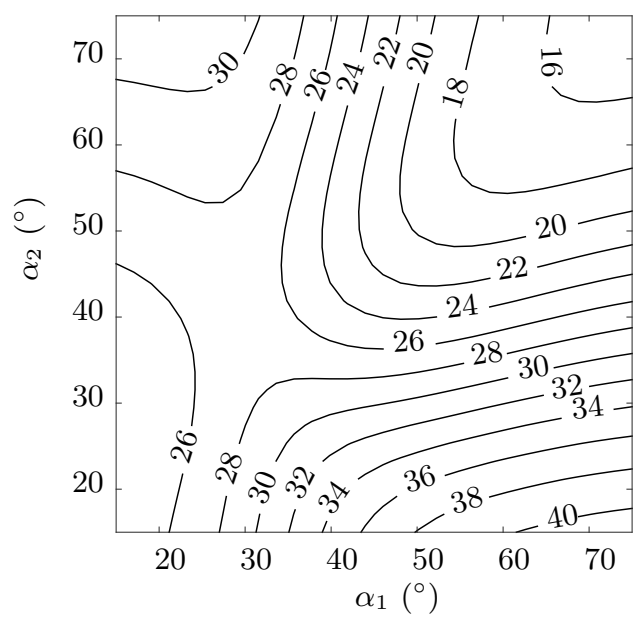

(b) Tube 5

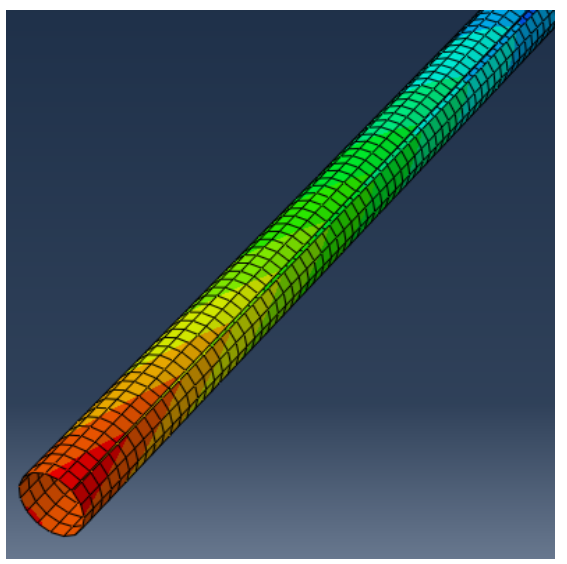

(d) Cross-section deformation of Tube 5

Figure 7: Frequency plots as a function of braid angle, and visualization of the tube cross-section deformation. The black lines in Figures (a), (b) and (c) represent the natural frequencies (Hz) involving the cantilever mode for Tube 4, Tube 5 and Tube 6 from Table 3; Figure (d) shows a (exaggerated) typical change in the shape of the cross-section of the free end of Tube 5 due to the transverse bending stiffness effect $[22,38]$. 
In the first instance, a constant longitudinal stiffness $A_{11}=1.0869 \times 10^{7} \mathrm{~N} / \mathrm{m}$ was selected to investigate the impact of $D_{11}$ on the natural frequency. Combinations of the braid angles $\alpha_{1}$ and $\alpha_{2}$ which keep $A_{11}$ constant were calculated using a Newton Raphson method, and these values were substituted into both the beam theory and the ABAQUS numerical model to calculate the cantilever frequency. The results, along with the braid angles $\alpha_{1}$ and $\alpha_{2}$, are plotted as functions of $D_{11}$ in Figure 8(a). In this plot, $D_{11}$ increases as $\alpha_{1}$ decreases and $\alpha_{2}$ increases. The frequency estimated by Equation 3 remains constant at $43.73 \mathrm{~Hz}$ due to the constant longitudinal stiffness, while the natural frequency computed in ABAQUS clearly varies substantially with $D_{11}$. The high disparity between beam theory prediction and FE simulation results indicates that simple beam theory is insufficient for frequency analysis of cantilevered extended BRC tubes and the cantilever frequency of BRC tubes is not only dependent on longitudinal stiffness.

Next, a constant flexural stiffness $D_{11}=0.0686 \mathrm{Nm}$ was chosen to investigate the impact of braid angles $\alpha_{1}$ and $\alpha_{2}$ on $A_{11}$, and the cantilever frequency. Numerical models with pairs of braid angles resulting in a constant $D_{11}$ were run in ABAQUS. The computed frequency and braid angles are plotted as functions of $A_{11}$ in Figure 8(b), in which $A_{11}$ increases with increasing $\alpha_{1}$ and decreasing $\alpha_{2}$. There is less variation in the $\alpha_{1}$ values than $\alpha_{2}$ calculated in this case. The frequency drops with increasing $A_{11}$ initially and then increases. This indicates that the flexural stiffness is also of importance in determining the dynamic response.

Finally, a constant coiled diameter of $65 \mathrm{~mm}$ was set to explore how braid angles $\alpha_{1}$ and $\alpha_{2}$ affect $A_{11}$, and the cantilever frequency. Braid angles and the lowest cantilever mode frequency computed in ABAQUS are plotted as a function of $A_{11}$ in Figure 8(c). It can be seen that $A_{11}$ increases with increasing $\alpha_{1}$ and decreasing $\alpha_{2}$. $D_{c}$ is a stronger function of $\alpha_{1}$ than $\alpha_{2}$, so there is less variation in the $\alpha_{1}$ values calculated in this case. The frequency decreases with increasing $A_{11}$ initially, and then increases. The highest frequency is achieved by reducing $\alpha_{2}$ to $\alpha_{\min }$ and calculating $\alpha_{1}$ so as to satisfy the $D_{c}$ constraint. This finding demonstrates that the highest natural frequency for this BRC tube is achieved by choosing minimum allowable braid angle for the inner ply within the laminate, which agrees with the nonlinear constraint vibration optimization results for Tube 7 from Table 5 . In addition, the coiled diameter shows significant sensitivity to the braid angles. This will be investigated further in Section 3.3. 


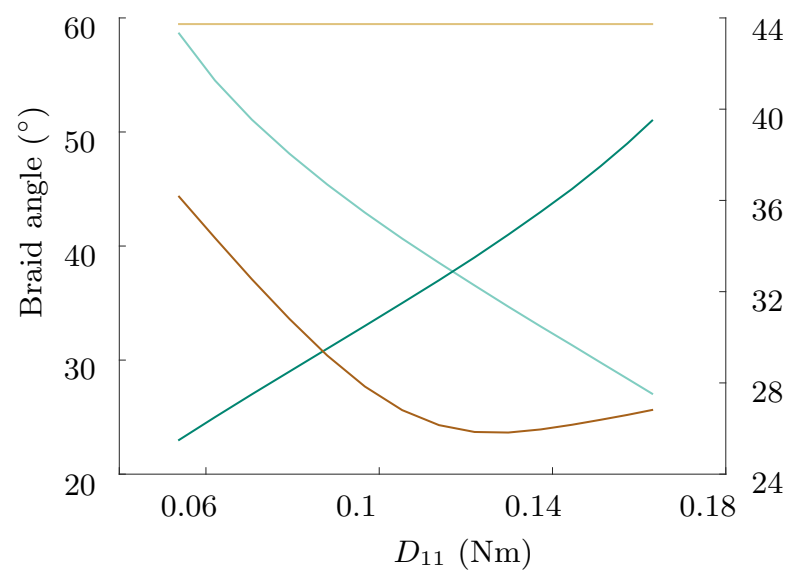

40 䨠

(a) $A_{11}$ constant at $1.0869 \times 10^{7} \mathrm{~N} / \mathrm{m}$

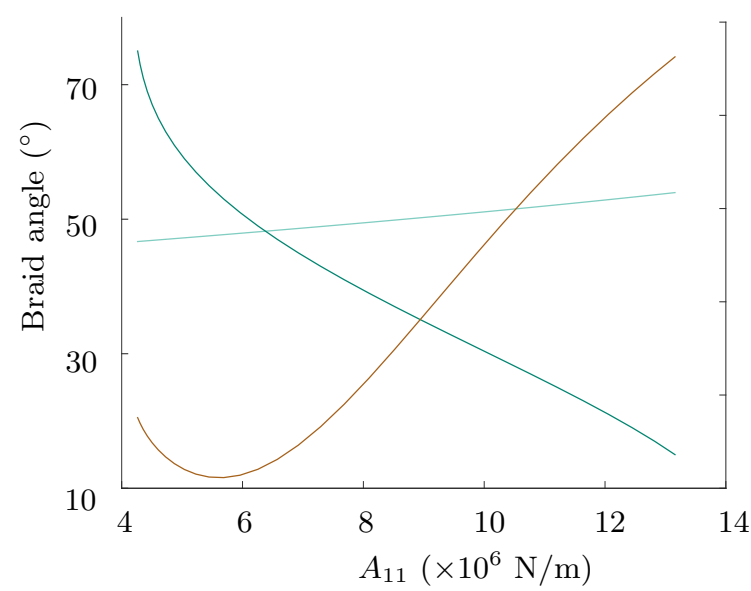

(b) $D_{11}$ constant at $0.0686 \mathrm{Nm}$

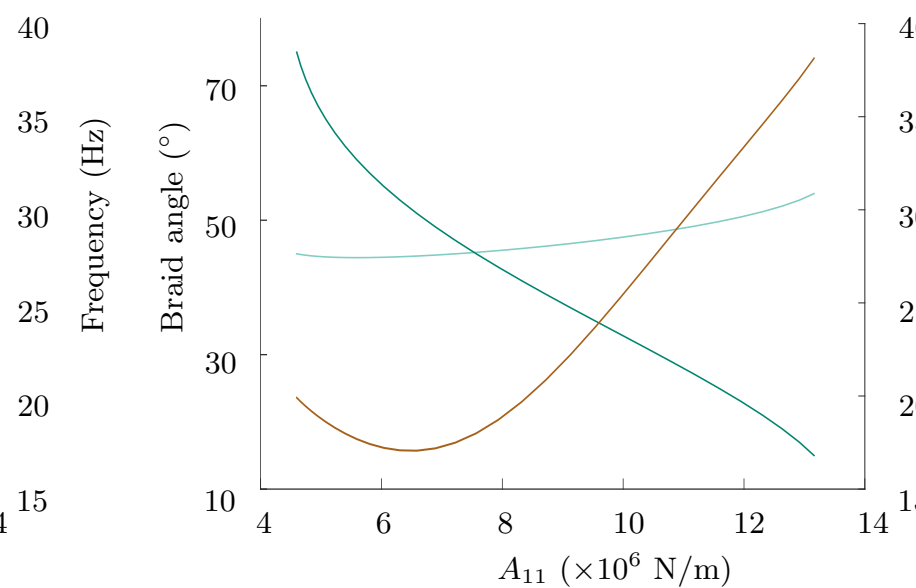

(c) $D_{c}$ constant at $65 \mathrm{~mm}$
Fequency (ABAQUS)

Frequency (Beam Theory)

$\alpha_{1}$

$\alpha_{2}$

Figure 8: Cantilever mode frequencies and braid angles as functions of key stiffness parameters for Tube 7 from Table 3. Figure (a) shows the natural frequencies and braid angles as functions of flexural stiffness $D_{11}$ at a constant longitudinal stiffness $A_{11}=1.0869 \times 10^{7}$ $\mathrm{N} / \mathrm{m}$. Figure (b) illustrates the frequency and braid angles as functions of $A_{11}$ at a constant $D_{11}=0.0686 \mathrm{Nm}$. Figure (c) shows the frequency and braid angles as functions of $A_{11}$ at a constant $D_{c}=65 \mathrm{~mm}$.

\subsection{Sensitivity of natural frequency and coiled diameter to braid angle perturbations}

Both dynamic response and coiled diameter are functions of ply angle within a BRC tube. It is often difficult to manufacture BRC tubes with fiber angles within $1^{\circ}$ (or even more) of the specified value. To evaluate the impact that manufacturing errors are likely to have on the coiled diameter and optimized frequency, a $1^{\circ}$ radius circle in $\alpha_{1} / \alpha_{2}$ space is traced around an optimal point and $D_{c}$ and the cantilever frequency evaluated along the path. The braid angles are set as:

$$
\begin{aligned}
& \alpha_{1}=\frac{\pi}{180} \cos \theta \\
& \alpha_{2}=\frac{\pi}{180} \sin \theta
\end{aligned} \quad \theta \in[0,2 \pi]
$$

The resulting frequency and $D_{c}$ variation for Tube 3 about the optimal braid angle point in Table 5 is shown in Figure 9. The circular $\alpha_{1}, \alpha_{2}$ path is traced about a stationary point in the frequency function, meaning that the variation in frequency to be expected due to a small manufacturing error is predictably small. However, the coiled diameter shows quite a large sensitivity to manufacturing errors in this region. This finding implies that the coiled 
diameter is more susceptible to braid angle errors than frequency is at an optimal point. The prescribed coiled diameter is likely to be constrained by the deployment mechanism or its housing, meaning particular care must be taken during the manufacture of frequency optimised BRCs.

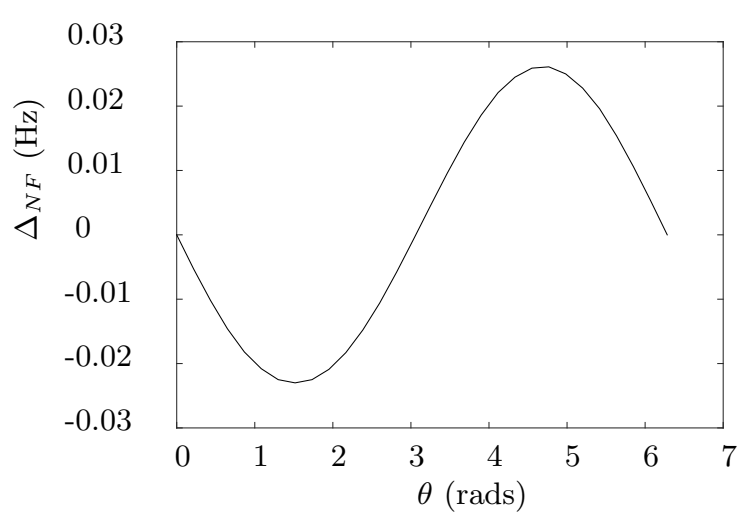

(a) Natural frequency

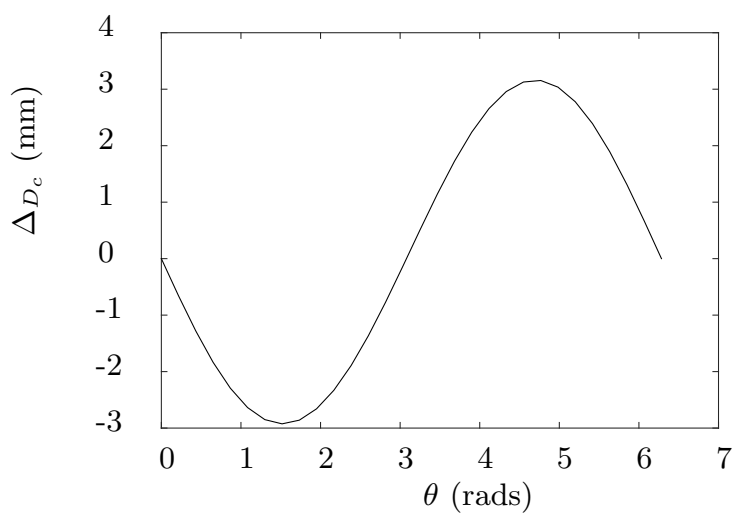

(b) Coiled diameter

Figure 9: Sensitivity analysis of frequency and coiled diameter of Tube 3 from Table 5 to $1^{\circ}$ radius circle in $\alpha_{1} / \alpha_{2}$ space traced around an optimal point. Figure (a) shows little variation in frequency because the circle origin is a stationary point in the frequency function. Figure (b) plots the likely variation of coiled diameter due to the $1^{\circ}$ error in braid angles, which implies that the coiled diameter is a strong function of braid angle at this location.

\section{Conclusion}

BRC tubes with different laminate stacking sequences and a deployed solar array without attached membrane cells were modeled in ABAQUS. The computed natural (cantilever mode) frequency, coiled diameter and stability margin were plotted as functions of braid angles over the allowable braid angle range. Great similarity of these contour plots confirms the feasibility of analyzing an individual tube instead of a complete solar array structure. The cantilever frequency contours for long slender BRC tubes are symmetric, meaning the ply locations within the laminate stack have little effect on the lowest cantilever frequency of these extended BRC tubes. Further analysis of the frequency contours for BRC tubes with different geometric properties shows asymmetry for short and deep tubes. A study of swapping the locations of braid plies within different tubes was also undertaken. An approach to optimizing the cantilever mode frequency of extended BRC tubes subject to stability and coiled diameter constraints by carefully selecting the braid angles has been formulated. It was discovered that in the majority of the cases analyzed, at least one of the constraints was active at the optimal point. Longitudinal and flexural stiffness of a bistable composite laminate have been investigated. The findings demonstrate the effects of flexural stiffness on the cantilever frequency of BRC tubes and the inaccuracies inherent in only accounting for the longitudinal stiffness in predicting the frequency using Euler beam theory. Sensitivity of coiled diameter and cantilever frequency to braid angle perturbations suggests extra care in keeping braid angles precise during manufacture should be taken.

\section{Acknowledgments}

The authors would like to acknowledge the technical and financial support from RolaTube Technology Ltd., the European Commission Framework 7 Program project DeployTech (project reference 284474) and the EPSRC (iCASE award). The authors confirm that the data underlying the findings are available without restriction. Details of the data and how to request access are available from University of Surrey publications repository: (http://epubs.surrey.ac.uk). 


\section{References}

[1] K. Iqbal, S. Pellegrino, A. Daton-Lovett, Bi-stable composite slit tubes, in: IUTAM-IASS Symposium on deployable structures: Theory and Applications, Springer, 2000, pp. 153-162.

[2] D. Campbell, R. Barrett, M. S. Lake, L. Adams, E. Abramson, M. Scherbarthn, J. S. Welsh, G. Freebury, N. Beidleman, J. Abbot, Development of a novel, passively deployed roll-out solar array, in: IEEE Aerospace Conference, IEEE, 2006.

[3] A. Viquerat, M. Schenk, M. Straubel, G. Secheli, M. Hillebrandt, M. Shahid, Report on modelling and simulation, EC Framework Programme 7 Deliverable 1.3 for DeployTech (project reference 284474), University of Surrey (2013).

[4] C. Wu, A. Viquerat, G. Aglietti, Natural frequency optimization and stability analysis of bistable carbon fiber reinforced plastic booms for space applications, in: 3rd AIAA Spacecraft Structures Conference, 2016.

[5] A. Daton-Lovett, Report on deployable mechanism design, EC Framework Programme 7 Deliverable 2.2 for DeployTech (project reference 284474), Rolatube Technology (2013).

[6] C. Bert, Optimal design of a composite-material plate to maximize its fundamental frequency, Journal of Sound and Vibration 50 (1977) 229-237.

[7] H. Fukunaga, H. Sekine, M. Sato, Optimal design of symmetric laminated plates for fundamental frequency, Journal of Sound and Vibration 171 (1994) 219-229.

[8] M. Miki, Material design of composite laminates with required in-plane elastic properties, in: Proc Progress in Science and Engineering of Composites, ICCM IV, Tokyo, 1982.

[9] M. Miki, Design of laminated fibrous composite plates with required flexural stiffness, in: Recent Advances in Composites in the United States and Japan, ASTM International, 1985.

[10] Y. Narita, Layerwise optimization for the maximum fundamental frequency of laminated composite plates, Journal of Sound and Vibration 263 (2003) 1005-1016.

[11] Y. Narita, J. Hodgkinson, Layerwise optimisation for maximising the fundamental frequencies of pointsupported rectangular laminated composite plates, Composite Structures 69 (2005) 127-135.

[12] D. N. Betts, H. A. Kim, C. R. Bowen, Optimization of stiffness characteristics for the design of bistable composite laminates, AIAA Journal 50 (2012) 2211-2218.

[13] A. W. Leissa, Vibration of shells, Vol. 288, Scientific and Technical Information Office, National Aeronautics and Space Administration Washington, DC, USA, 1973.

[14] K. Seffen, S. Pellegrino, Deployment dynamics of tape springs, Proceedings of the Royal Society of London. Series A: Mathematical, Physical and Engineering Sciences 455 (1999) 1003-1048.

[15] W. Soedel, Vibrations of shells and plates, CRC Press, 2004.

[16] N. Carrère, Y. Rollet, V. Retel, L. Boubakar, J.-F. Maire, Composites structural modelling with uncertain data, Composites Science and Technology 69 (2009) 60-66.

[17] C. Scarth, P. Sartor, J. Cooper, P. M. Weaver, G. H. Siva, Robust aeroelastic optimisation of composite plate wings subject to ply orientation uncertainty, in: 20th International Conference on Composite Materials, 2015.

[18] C. Scarth, J. Cooper, P. M. Weaver, G. H. Siva, Uncertainty quantification of aeroelastic stability of composite plate wings using lamination parameters, Composite Structures 116 (2014) 84-93.

[19] C. J. Brampton, D. N. Betts, C. R. Bowen, H. A. Kim, Sensitivity of bistable laminates to uncertainties in material properties, geometry and environmental conditions, Composite Structures 102 (2013) 276-286. 
[20] S. Sriramula, M. K. Chryssanthopoulos, Quantification of uncertainty modelling in stochastic analysis of FRP composites, Composites Part A: Applied Science and Manufacturing 40 (2009) 1673-1684.

[21] L. Mehrez, A. Doostan, D. Moens, D. Vandepitte, Stochastic identification of composite material properties from limited experimental databases, Part II: Uncertainty modelling, Mechanical Systems and Signal Processing 27 (2012) 484-498.

[22] L. S. Cecchini, P. M. Weaver, Optimal fiber angles to resist the brazier effect in orthotropic tubes, AIAA Journal 40 (2002) 2136-2138.

[23] A. Brinkmeyer, S. Pellegrino, P. M. Weaver, Effects of long-term stowage on the deployment of bistable tape springs, Journal of Applied Mechanics 83 (1) (2016) 011008-1-11.

[24] D. Jiang, Y. Li, Q. Fei, S. Wu, Prediction of uncertain elastic parameters of a braided composite, Composite Structures 126 (2015) 123-131.

[25] T. G. Ritto, R. Sampaio, Stochastic drill-string dynamics with uncertainty on the imposed speed and on the bit-rock parameters, International Journal for Uncertainty Quantification 2 (2) (2012) 111-124.

[26] C. Wu, A. Viquerat, G. Aglietti, Improving the natural frequency of bistable carbon fibre reinforced plastic tubes for space applications, Journal of the International Association for Shell and Spatial Structures 56 (2015) 259-267.

[27] Dassault Systèmes, Abaqus 6.14.

URL http://www.3ds.com/products-services/simulia/products/abaqus

[28] The MathWorks, Inc., Matlab 2015a.

URL http://uk. mathworks .com/products/matlab/?requestedDomain=www . mathworks . com

[29] A. T. Nettles, Basic mechanics of laminated composite plates, Marshall Space Flight Center, National Aeronautics and Space Administration, 1994.

[30] S. Guest, S. Pellegrino, Analytical models for bistable cylindrical shells, Proceedings of the Royal Society A: Mathematical, Physical and Engineering Science 462 (2006) 839-854.

[31] J. M. Fernandez, A. Viquerat, V. J. Lappas, A. J. Daton-Lovett, Bistable over the whole length (BOWL) CFRP booms for solar sails, in: Advances in Solar Sailing, 2014, pp. 609-628.

[32] E. A. Thornton, Y. A. Kim, Thermally induced bending vibrations of a flexible rolled-up solar array, Journal of Spacecraft and Rockets 30 (1993) 438-448.

[33] Manual, Abaqus users. version 6.14-1, Dassault Systémes Simulia Corp., Providence, RI.

[34] Z. Wu, G. Raju, S. White, P. M. Weaver, Optimal design of postbuckling behaviour of laminated composite plates using lamination parameters, in: 55th AIAA/ASME/ASCE/AHS/ASC Structures, Structural Dynamics, and Materials Conference, AIAA SciTech, 2014.

[35] A. L. Custodio, L. N. Vicente, SID-PSM: A pattern search method guided by simplex derivatives for use in derivative-free optimization (2008).

[36] J. M. Fernandez, Low-cost gossamer systems for solar sailing \& spacecraft deorbiting applications, Ph.D. thesis, University of Surrey (2014).

[37] J. S. Tate, A. D. Kelkar, J. D. Whitcomb, Effect of braid angle on fatigue performance of biaxial braided composites, International Journal of Fatigue 28 (2006) 1239-1247.

[38] L. S. Cecchini, P. M. Weaver, Brazier effect in multibay airfoil sections, AIAA Journal 43 (2005) 2252-2258.

[39] R. Irwin, J. Veen, E. Buchner-Santos, C. Dharan, Low-mass deployable spacecraft booms, in: AIAA SPACE Conference and Exposition, 2010. 